\title{
SDG711 Is Involved in Rice Seed Development through Regulation of Starch Metabolism Gene Expression in Coordination with Other Histone Modifications
}

\author{
Xiaoyun Liu ${ }^{1 *+} \mathbb{O}$, Junling Luo ${ }^{3 \dagger}$, Tiantian $\mathrm{Li}^{4}$, Huilan Yang ${ }^{1}$, Ping Wang ${ }^{1}$, Lufang Su${ }^{1}$, Yu Zheng ${ }^{1}$, Chun Bao and \\ Chao Zhou ${ }^{2^{*}}$
}

\begin{abstract}
SDG711 is a histone H3K27me2/3 transmethylase in rice, a homolog of CLF in Arabidopsis, and plays key roles in regulating flowering time and panicle development. In this work, we investigated the role of SDG711 in rice seed development. Overexpression and downregulation of SDG711 lead to a decrease and increase in the expression level of genes related to starch accumulation, resulting in smaller seeds or even seed abortion. ChIP assay showed that SDG711-mediated H3K27me3 changed significantly in genes related to endosperm development, and SDG711 can directly bind to the gene body region of several starch synthesis genes and amylase genes. In addition, H3K4me3 and H3K9ac modifications also cooperate with H3K27me3 to regulate the development of the endosperm. Our results suggest that the crosstalk between SDG711-mediated H3K27me3 and H3K4me3, and $\mathrm{H} 3 \mathrm{~K} 9 \mathrm{ac}$ are involved in starch accumulation to control normal seed development.
\end{abstract}

Keywords: Rice, SDG711, Starch accumulation, Cooperation, Histone methylation and acetylation

\section{Background}

Rice is one of the most important alimentary crops and provides food for more than half of the world's population. The main body of the rice seed is the food. Starch metabolism is a key process in seed development and directly affects grain yield and quality. Starch metabolism mainly comprises starch synthesis and starch degradation.

\footnotetext{
*Correspondence: Ixytcw@webmail.hzau.edu.cn; 18995654496@163.com; zhouchao@ctgu.edu.cn

${ }^{+} X i a o y u n$ Liu and Junling Luo contributed equally to this work. ${ }^{1}$ Institute for Interdisciplinary Research, Jianghan University, Wuhan 430056, China

${ }^{2}$ Key Laboratory of Three Gorges Regional Plant Genetics \& Germplasm Enhancement (CTGU) /Biotechnology Research Center, China Three Gorges University, Yichang 443002, China

Full list of author information is available at the end of the article
}

Starch synthesis genes in the seed can be divided into four groups according to the specific tissue and developmental stage: group I genes, which are expressed during the very early periods of grain formation and are thought to be involved in the construction of fundamental cell machinery and initiation of starch granules; group II genes, which are highly expressed during endosperm development; group III genes, of which transcript levels are low at the onset but rise sharply at the start of starch synthesis in the endosperm and are presumed to play essential roles in endosperm starch synthesis; and group IV genes, which are mainly expressed at the onset of grain development with scanty expression and might be involved in synthesis of starch in the pericarp (Ohdan et al. 2005). The mechanism regulating starch biosynthesis in cereal seeds is not well understood. To date, genetic 
and functional genomics studies have only identified some key regulators of starch synthesis. For example, the MYC transcriptional factor OsBP-5 can interact with an ethylene-responsive element binding protein (EREBP), OsEBP-89, to enhance the transcription of OsGBSSI which belongs to the group III starch synthesis genes. Knockdown of OsBP-5 expression results in reduced expression of OsGBSSI, leading to a reduction in the amylose content of mature seeds (Zhu et al. 2003). The FLO2 gene, encoding a nuclear-localized TPR-binding protein, has been shown to positively regulate the expression of starch synthesis-associated genes by interacting with bHLHs transcription factors (She et al. 2010). The AP2/EREBP TF gene RSR1 negatively regulates starch synthesis genes including OsGBSSI, OsSSI and OsSSIIIa, In rsr 1 mutants, the expression levels of these genes are increased, while in RSR1 overexpression, the expression of these genes is decreased (Fu and Xue 2010). It has been shown that OsbZIP58 encodes a key transcriptional regulator required for starch synthesis through directly binding to the promoters of OsAGPL3, OsGBSSI, OsSSIIa, SBE1, OsBEIIb, and ISA2 to promote their expression. (Wang et al. 2013). During grain filling, starch biosynthesis genes such as OsGBSSI, OsSSI, OsSSIIIa, and OsAGPL2 are up-regulated in SERF1 knockout grains. Moreover, SERF1 is a direct upstream regulator of GBSSI (Schmidt et al. 2014). In contrast to these positive and negative regulators controlling starch biosynthesis gene expression, FLO6 (a CBM domain containing protein) may act as a starch-binding protein involved in starch synthesis and compound starch grain formation through direct interaction with isoamylase1 (OsISA1) which belongs to the third group of starch synthase genes in developing rice seeds (Peng et al. 2014). In addition to genetic approaches, genome-wide DNA methylation analysis of a series of developmental stages of rice endosperm has revealed that DNA methylation is involved in the repression of genes involved in starch synthesis during seed development (Xing et al. 2015).

On the other hand, starch degradation genes mainly contain $\alpha$ - and $\beta$-amylases (Asatsuma et al. 2006; Zhang et al. 2016). There are few studies on the regulation mechanism of amylases. It has been shown that SERF1 negatively regulates germination by controlling $R P B F$ expression, which mediates the gibberellic acid (GA)-induced expression of RICE AMYLASE1A (RAmy1A). Loss of $S E R F 1$ enhances RPBF expression, resulting in larger grains with increased starch content, while SERF1 overexpression represses $R P B F$, resulting in smaller grains (Schmidt et al. 2014). OsSRT1 mediates H3K9ac by directly binding to starch metabolism genes such as OsAmy3B, OsAmy3E, OsBmy4, and OsBmy9 to regulate the expression of these genes, and down-regulation of OsSRT1 leads to abnormal seed development (Zhang et al. 2016). Therefore, the regulation of starch metabolism is crucial for determining rice yield and quality. However, the chromatin and epigenetic mechanisms that directly regulate rice starch metabolism are not well described.

Histone acetylation and methylation are important chromatin modifications that regulate gene expression during plant development (He et al. 2011). In particularly, H3 lysine 9 acetylation (H3K9ac) and $\mathrm{H} 3$ lysine 4 trimethylation (H3K4me3) are closely associated with genes with high expression levels and low tissue specificity, whereas histone $\mathrm{H} 3$ lysine 27 trimethylation (H3K27me3) is associated with genes with low expression levels and high tissue specificity. (Charron et al. 2009; Makarevitch et al. 2013; Zhang et al. 2009; Zhang et al. 2007). Polycomb group (PcG) and Trithorax-group (TrxG) proteins catalyze H3K27me3 or H3K4me3, respectively (Steffen and Ringrose 2014). Several studies have revealed that some development-related genes have bivalent modifications (H3K4me3-H3K27me3) to coordinately regulate their expression in plants (Berr et al. 2010; Sequeira-Mendes et al. 2014). Our previous study also suggested that the dynamic change in H3K27me3/ H3K4me3 ratio of bivalent marking genes related to development during the SAM-to-IM transition is critical for genome-wide gene expression reprogramming in IM (Liu et al. 2015). Subsequently, readers (EBS and SHL) were identified as having the ability to recognize both H3K27me3 and H3K4me3 via their bromo-adjacent homology (BAH) and plant homeodomain (PHD) domains, which further confirmes that the genes can be coordinately regulated by different histone modifications (Qian et al. 2018; Yang et al. 2018).

Polycomb-repressive complex 2 (PRC2), a subset of the PcG proteins, has four core proteins: ENHANCER OF ZESTE $[\mathrm{E}(\mathrm{z})], \quad$ SUPPRESSOR OF ZESTE 12 [Su(z)12], EXTRA SEXCOMBS (ESC) and P55 (Schuettengruber and Cavalli 2009). The $\mathrm{E}(\mathrm{z})$ protein catalyzes the addition of H3K27 methylation (Czermin et al. 2002). There are three $E(z)$ homologs that catalyze the addition of H3K27me3 in Arabidopsis: CURLY LEAF (CLF), SWINGER (SWN), and MEDEA (MEA). MEA plays an important role in gametogenesis and early seed development, whereas CLF/SWN are partially redundant and function primarily in vegetative and reproductive development (Hennig and Derkacheva 2009). Homologs of E(z) (OsCLF/SDG711 and OsiEZ1/SDG718) have also been found in rice (Luo et al. 2009). They are required for $\mathrm{H} 3 \mathrm{~K} 27 \mathrm{me} 3$ during rice flowering development and reproductive transition (Liu et al. 2014b; Liu et al. 2015). They may be involved in regulating seed dormancy, seedling growth, vegetative and reproductive development and seed development together with other PRC2 components in rice (Chen et al. 2017; Huang et al. 2016; Li et al. 2014; Liu et al. 2016; Nallamilli et al. 2013; Zhong et al. 2018). Previous studies have shown that 
during rice seed development, PRC2 complex mainly regulates the development of the embryo, endosperm, and seed coat by regulating transcription factors (Huang et al. 2016; Nallamilli et al. 2013).

In this study, we showed that both SDG711 RNAi plants and overexpression plants produced smaller seeds. To understand the molecular mechanism, we examined the expression and histone modification of starchrelated genes, and the direct association of SDG711 with those genes by ChIP assay using anti-SDG711 antibody. Our results suggest that SDG711 directly represses the expression of several starch synthesis genes and amylase genes through H3K27me3 modification, leading to impairing starch accumulation in developing seeds. In addition, H3K4me3 and H3K9ac enrichment also changed on these target genes. Our results suggest that H3K27me3 and H3K4me3 have antagonistic effects on starch synthesis genes and H3K27me3 and H3K9ac have antagonistic effects on amylase genes, respectively. The cooperation of SDG711-mediated H3K27me3 with H3K4me3 and H3K9ac is involved in starch accumulation to regulate normal seed development.

\section{Materials and Methods}

\section{Plant Materials and Growing Conditions}

Rice (Oryza sativa spp japonica) material used in this study was from the 'DongJin' (DJ) background, including the wild type (WT), SDG711 (OsCLF) overexpression, and RNAi lines (Liu et al. 2014b). The germinated rice seedlings of all genotypes were transplanted in the field at the beginning of May and grown till the middle of August in Wuhan. To analyze developmental seeds, spikelet samples were collected daily from 1 day after pollination (DAP) to $7 \mathrm{DAP}$. The flowering spikelets were tagged using a pen mark on the lemmas. Approximately 300 developing seeds from 30 rice plants of each line were collected, and three biological repetitions were performed.

\section{Microscopy Analysis of Endosperm Structure}

The collected spikelet samples were immediately fixed in $5 \%(\mathrm{v} / \mathrm{v})$ formaldehyde, $5 \%(\mathrm{v} / \mathrm{v})$ acetic acid, $45 \%(\mathrm{v} / \mathrm{v})$ ethanol, and $45 \%(\mathrm{v} / \mathrm{v})$ distilled, deionized water at $4{ }^{\circ} \mathrm{C}$, followed by vacuum infiltration until the samples sank to the bottom of the container. Fixed samples were embedded using a Technovit 7100 (Heraeus Kulzer) and then cut into semi-thin sections of $1-5 \mu \mathrm{m}$ thickness using a Leica RM2265 microtome for imaging.

\section{Gene Expression Analysis}

Total RNA was extracted from seeds of 3DAP using TRIzol reagent (TransGen Biotech) according to the manufacturer's protocol. Two micrograms of total RNA were reverse-transcribed to obtain cDNA by using
DNase I and M-MLV Reverse Transcriptase (Invitrogen) according to the manufacturer's instructions. Synthesized first-strand cDNA was used as a template for qRT-PCR. qRT-PCR was performed on an ABI 7900 instrument. The reactions were performed at $95^{\circ} \mathrm{C}$ for $10 \mathrm{~s}$, then 45 cycles of $95^{\circ} \mathrm{C}$ for $5 \mathrm{~s}$ and $60^{\circ} \mathrm{C}$ for $40 \mathrm{~s}$. The disassociation curve analysis was performed as follows: $95^{\circ} \mathrm{C}$ for $15 \mathrm{~s}, 60^{\circ} \mathrm{C}$ for $20 \mathrm{~s}$, and $95^{\circ} \mathrm{C}$ for $15 \mathrm{~s}$. Data were collected using the ABI 7900 sequence detection system following the manufacturer's instructions. Relative expression levels were analyzed using the $2^{-\triangle \triangle C T}$ method (Livak and Schmittgen 2001). The rice ACTIN1 gene was used as an internal control. The primers shown in Table S1 were designed using PRIMER EXPRESS 2.0 software (PE Applied Biosystems) to amplify $80-250$ bp products.

\section{ChIP and Re-ChIP Assay}

ChIP analysis was performed as previously described $(\mathrm{Hu}$ et al. 2012). Briefly, chromatin isolated from $4 \mathrm{~g}$ of seeds at 3 DAP was incubated with antibody-coated beads (Life technology, 10001D) overnight. After washing and elution, the products were reverse cross-linked. The products were then treated with protease K (9034, Takara), recovered, and used as a template for real-time PCR with primers listed in Table S1. Antibodies used for histone modifications were anti-H3K4me3 (ab8580, Abcam), anti-H3K27me3 (07-449, Millipore) and anti-H3K9ac (07-352, Millipore) respectively. The Antibody of SDG711 was produced by immunizing rabbits with E.coli produced full-length SDG711 protein (Liu et al. 2014b).

Tissue fixing, chromatin sonication, and IP with the first antibody in the primary ChIP procedure were performed as Described above. The first antibody was crosslinked to the beads using the fixative disuccinimidyl suberate (DSS, Pierce, 21,555). After washing with the buffers, the protein-DNA complexes were eluted from beads by ChIP Elution buffer (50mMTris- $\mathrm{HCl} \mathrm{pH} 7.5$, $10 \mathrm{mM}$ EDTA, $1 \%$ SDS) for $15 \mathrm{~min}$ at $65^{\circ} \mathrm{C}$. The eluted chromatin was then used for the reChIP. ReChIP with the secondary antibody, elution, DNA purification were performed as described above.

\section{Yeast Two-Hybrid Assay}

Constructs for yeast two-hybrid analysis were generated using the Matchmaker ${ }^{\circ}$ Gold Yeast Two-Hybrid System (Clontech) vectors pGBKT7 and pGADT7, which express protein fusions to the GAL4 DNA-binding domain or transcriptional-activation domain, respectively. Full-length of cDNA inserts encoding OsCLF (SDG711) and OsiZE1 (SDG718) were introduced into pGADT7, and full-length of cDNA inserts encoding OsiZE1, OsFIE1, OsFIE2 and OsEMF2b were introduced into pGBKT7. The analysis was performed in strain AH109 carrying HIS3 and MEL1 reporters for reconstituted GAL4 activity. 


\section{Starch Contents, Protein Content and 100-Grain Weight Determination}

Fully filled grains of 30 DAP were used to measure grain quality and yield traits. The embryo and pericarp were removed from the dehulled grains, and the endosperm was ground to powder. The apparent amylose content (AAC) of the sample was measured by the iodine colorimetric method (Juliano 1971). To determine the total starch content, $50 \mathrm{mg}$ of powder was washed two to three times using $80 \%(\mathrm{v} / \mathrm{v})$ ethanol and then subjected to extraction using 9.2 and $4.6 \mathrm{M}$ perchloric acid. The supernatant was collected and diluted to $50 \mathrm{~mL}$ with water. An aliquot of this solution was analyzed for starch content by the anthrone method (Turner and Turner 1960). Seed storage protein content was measured using near-infrared reflectance using an XDS Rapid Liquid Analyzer (FOSS Tecator AB, Sweden). All samples were measured using three replicates (Ge et al. 2007). The 100 -grain weight was determined by counting 10 replicates of 100-grain samples independently on an electronic balance. Data are shown as the mean \pm SD.

\section{Results}

Knockdown and Overexpression of the SDG711 Gene Leads to Abnormal Seed Development in Rice

Our previous studies characterized transgenic and mutant plants for the rice $E(Z)$ gene $S D G 711$ and showed that SDG711 regulates rice flowering time and inflorescence meristem activity (Liu et al. 2014b; Liu et al. 2015). All phenotypic statistics come from three parallel lines, and all molecular biology experiments have been performed a mixture of three lines. In this study, we also observed that SDG711 expression levels affect rice seed development. Seed setting rate was reduced by $71-89 \%$ compared to WT in both overexpression and RNAi plants (Figure S1).
Through the investigation of the structure of the flowers, it was shown that the stigmas and anthers were not significantly different from WT in the external structure, whether in overexpression plants or RNAi plants. Observation of the morphology of seeds at different days after fertilization shows that seeds of overexpression plants developed significantly more slowly than WT seeds, and most of them did not develop into full seeds normally (Figure S1). Subsequently, the agronomic traits of overexpression, RNAi, and WT plants in fully mature seeds were measured. Compared with WT, the grain width and 100-grain weight of overexpression and RNAi plants were significantly decreased (Fig. 1). This indicates that SDG711 may be involved in the very complicated regulation pathways of the seed development process.

\section{SDG711 Affects Starch Accumulation in Endosperm Cells at the Early Stage of Seed Development}

Next, we examined the development of the internal structure of the seeds using semi-thin sections. The cross sections of 3 and 4 DAP seeds showed that the number of starch granules in the endosperm of overexpression plants and RNAi plants was much lower than in WT plants. In addition, the process of starch accumulation was hindered, and the starch granules are loosely distributed and cannot completely fill the cavity in the middle of the endosperm (Fig. 2a). In order to identify the mechanism related to decreased seed quality, we measured the total starch content and apparent amylose content (AAC) in mature seeds of overexpression, RNAi, and WT plants. The measurements showed that the starch content of seeds in overexpression and RNAi plants were significantly reduced by $16-19 \%$ compared to that in WT plants, while the ACC contents were reduced by $40-52 \%$ compared to that in WT plants
A

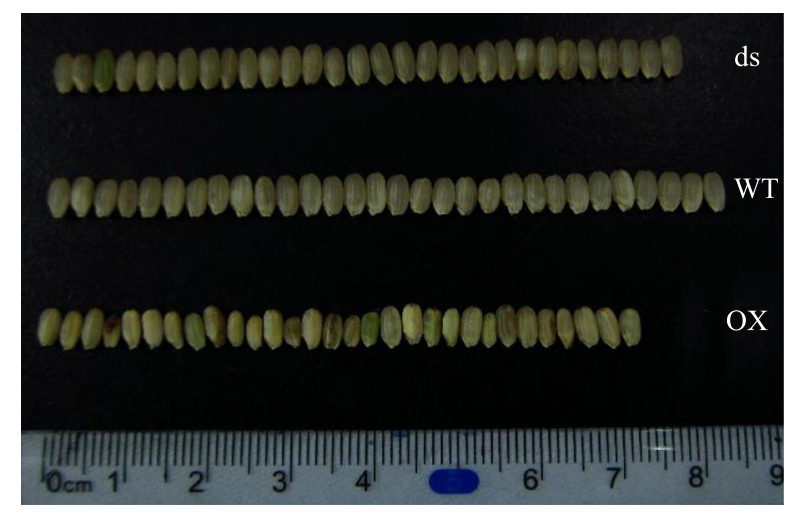

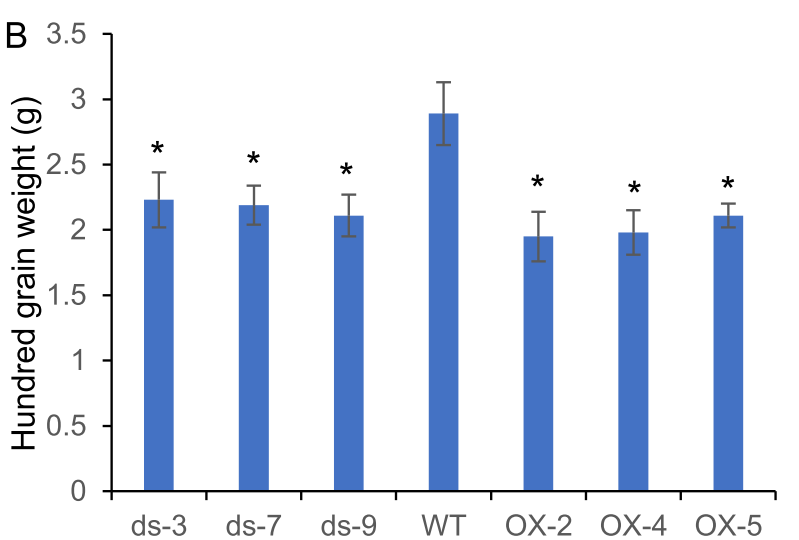

Fig. 1 Phenotypic analysis of SDG711 transgenic seeds. a. Smaller mature seeds of SDG711 RNAi and overexpression transgenic plants. Bars $=0.5$ $\mathrm{cm}$. ds indicates independent lines of SDG711 RNAi transgenic plants; OX indicates independent lines of SDG711 overexpression transgenic plants. b. The 100-grain weight of SDG711 RNAi, overexpression transgenic plants, and wild type. * significantly different $(p<0.01)$. Values are mean \pm SD. ds3, ds-7 and ds-9 indicate independent lines of SDG711 RNAi transgenic plants; OX-2, OX-4, and OX-5 indicate independent lines of SDG711 overexpression transgenic plants 
A
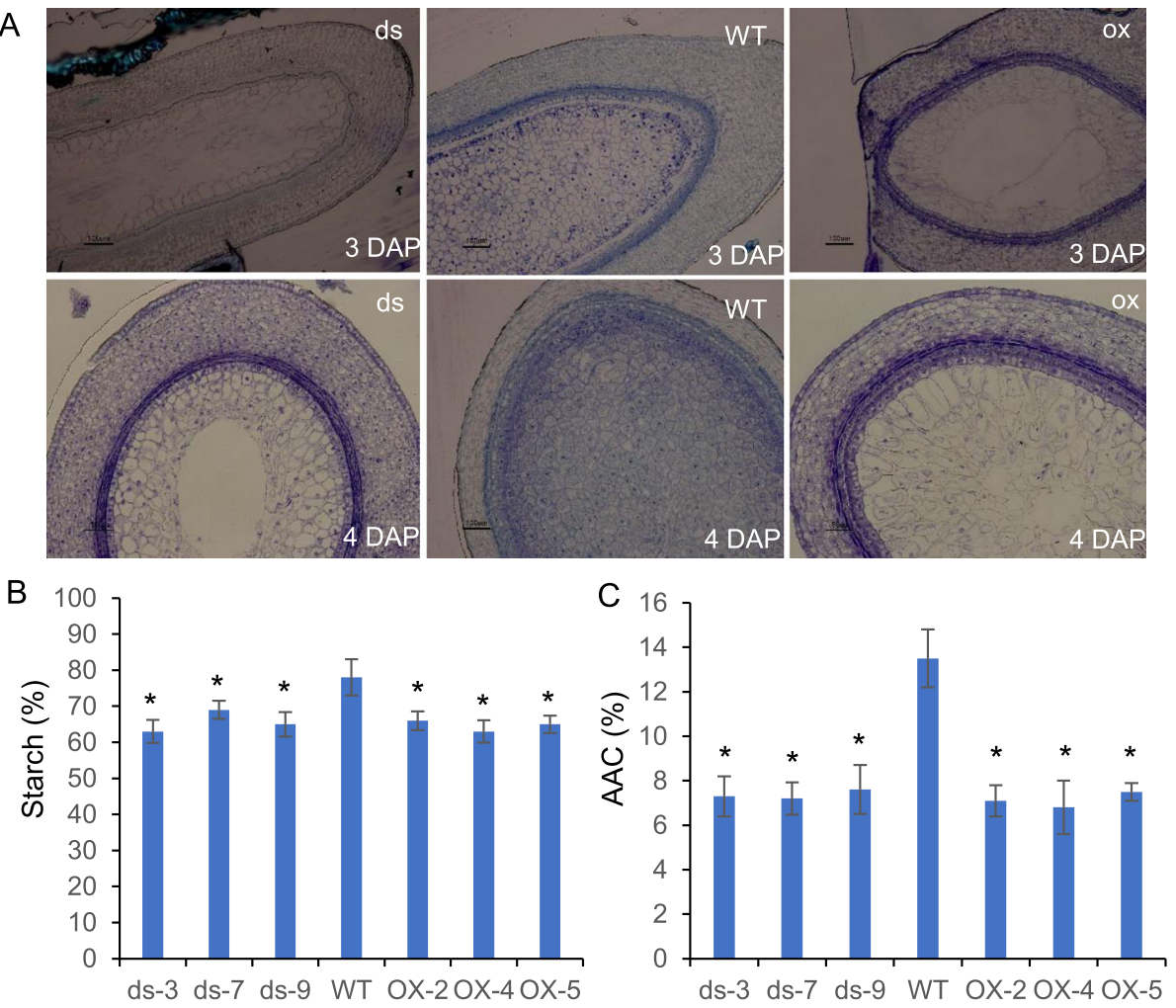

Fig. 2 Endosperm starch granules and starch content of SDG711 transgenic plants. a. Cross sections of seeds at 3 DAP and 4 DAP. ds indicates independent lines of SDG711 RNAi transgenic plants; OX indicates independent lines of SDG711 overexpression transgenic plants. $\mathbf{b}$. Total starch content in endosperm of SDG711 transgenic plants. c. AAC in endosperm of SDG711 transgenic plants. *significantly different $(p<0.01)$. Values are mean \pm SD. ds3, ds-7 and ds-9 indicate independent lines of SDG711 RNAi transgenic plants; OX-2, OX-4 and OX-5 indicate independent lines of SDG711 overexpression transgenic plants

(Fig. 2b). We also tested the content of the four main storage proteins (albumin, globulin, prolamin, and glutelin), and observed that the storage protein content in the seeds of overexpression and RNAi plants did not change significantly compared with those in WT plants (Figure S2). The above results suggest that SDG711 may regulate seed development by playing vital roles in starch accumulation and distribution, but not in protein storage in the endosperm.

\section{Starch Metabolism Related Genes Were Differentially Expressed in Overexpression and RNAi Lines}

The accumulation of starch in the seed endosperm is directly related to starch synthesis and starch degradation. In order to determine the causes of the low starch content in SDG711 overexpression and RNAi lines, we analyzed the expression levels of starch metabolism-related genes in the three DAP seeds, including regulators that affect starch metabolism, starch synthesis-related genes, and starch degradation-related genes. First, we tested the typical regulator genes RSR1, OsbZIP58, FLO2, FLO6, SERF1 and OsBP-5 which have been found to regulate starch metabolism. However, there were no obvious differences between SDG711 overexpression, RNAi lines, and WT plants (Figure S3). Next, we examined 24 genes of four groups of starch synthase (OsSSI, OsSSIIa, OsSSIIb, OsSSIIc, OsSSIIIa, OsSSIIIb, OsSSIVa, OsSSIVb, OsAGPS1, OsAGPS2a, OsAGPS2b, OsAGPL1, OsAGPL2, OsAGPL3, OsAGPL4, OsGBSSI, OsGBSSII, OsBEI, OsBEIIa, OsBEIIb, OsISA1, OsISA2, OsISA3, and OsPUL), 20 starch degradation genes including $10 \alpha$-amylase genes (OsAmy1A, OsAmy1B, OsAmy1C, OsAmy2A, OsAmy3A, OsAmy3B, OsAmy3D, OsAmy3E, OsAmy4A, and OsAmy5A), and 10 $\beta$-amylase genes (OsBmy1, OsBmy2, OsBmy3, OsBmy4, OsBmy5, OsBmy6, OsBmy7, OsBmy8, OsBmy9, and OsBmy10). The expression of 14 starch synthesis genes (OsSSI, OsSSIIa, OsSSIIc, OsSSIIIa, OsSSIVa, OsAGPS2b, OsAGPL2, OsAGPL3, OsGBSSI, OsBEI, OsBEIIb, OsISA1, OsISA2, and OsPUL) was higher in SDG711 RNAi than in WT seeds, and lower in SDG711 overexpression than in WT seeds. The degree of decrease in overexpression lines was greater than the degree of increase in RNAi lines (Fig. 3a). The expression of four $\alpha$-amylase genes (OsA$m y 1 C$, OsAmy3B, OsAmy3E, and OsAmy5A) and six $\beta$ amylase genes (OsBmy3, OsBmy4, OsBmy6, OsBmy7, OsBmy9, and OsBmy10) was also higher in SDG711 RNAi 

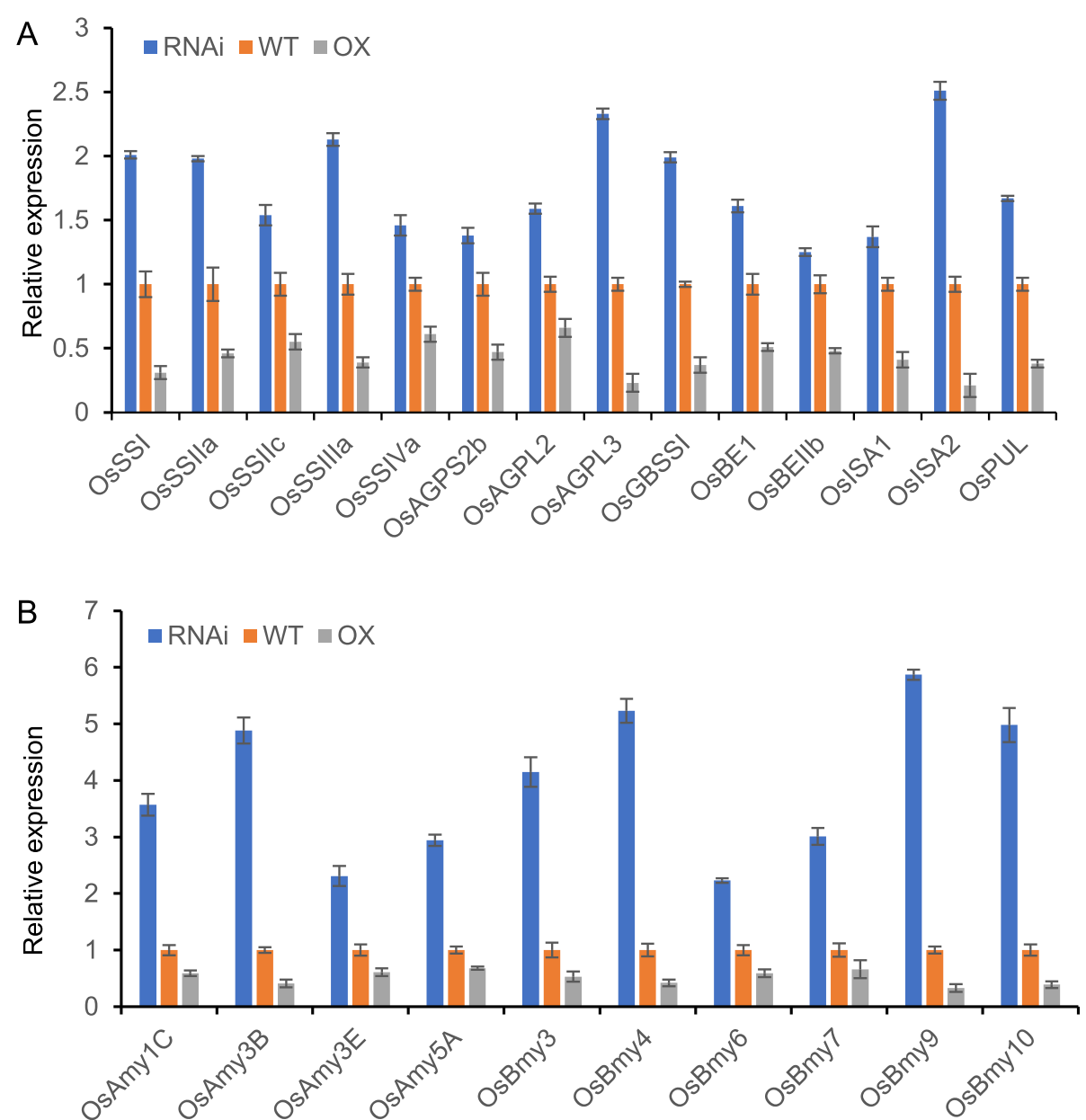

Fig. 3 Expression level of genes associated with starch metabolism in WT and SDG711 transgenic seeds at 3 DAP. Real-time PCR detection of transcript levels of starch synthase genes (a) and amylase genes (b) in WT and SDG711 transgenic seeds at 3 DAP. RNAi indicates lines of SDG711 RNAi transgenic plants and OX indicates lines of SDG711 overexpression transgenic plants. Each qRT-PCR assay was repeated three times. Values are mean $\pm \mathrm{SD}$. Values are shown relative to the ACTIN transcript levels

than WT seeds, and lower in SDG711 overexpression than in WT seeds. However, the degree of decrease in the overexpression lines was lower than the degree of increase in RNAi lines (Fig. 3b). Similar expression levels of other genes were detected in the overexpression, RNAi, and WT seeds (Figure S4). This result suggested that in the process of starch accumulation, the expression of starch synthesis genes was reduced by a greater extent than amylase genes, thus the starch synthesis genes may play a leading role in $S D G 711$ overexpressing lines, while the expression of the starch amylase genes was increased more than the starch synthesis genes, thus the starch amylase genes may play a leading role in SDG711 RNAi lines. Therefore, we can observe that the accumulation of starch in both overexpression and knockout plants is reduced.

Our previous study confirmed that SDG711 is a histone methyltransferase, that catalyzes the addition of H3K27me3 (Liu et al. 2014b). Changes in SDG711 expression can affect genome-wide H3K27me3 in rice (Liu et al. 2015). In order to study whether changes in the expression level of SDG711 would affect histone modification changes on these starch-metabolizing enzymes, ChIP assay was performed to analyze H3K27me3 on these genes in overexpression, RNAi, and WT seeds at 3 DAP. Given that H3K27me3 was enriched within gene bodies, mostly at the $5^{\prime}$ end of the rice genes, we analyzed ChIP by real-time PCR using two primer sets, one corresponding to the $5^{\prime}$ transcriptional start site (TSS), and the other to the $5^{\prime}$ end of the gene body (Fig. 4). Among these genes, six starch synthesis genes (OsSSI, OsSSIIa, OsAGPL3, OsGBSSI, OsBEI, and OsISA2) and four starch amylase genes (OsAmy1C, OsAmy3B, OsBmy4, and OsBmy9) displayed H3K27me3 in the gene body region, suggesting that regulation of these genes might involve PRC2 function. H3K27me3 on these genes was clearly reduced in the RNAi lines but increased in the overexpression lines (Fig. 4), which inversely correlated with their expression changes in the transgenic 


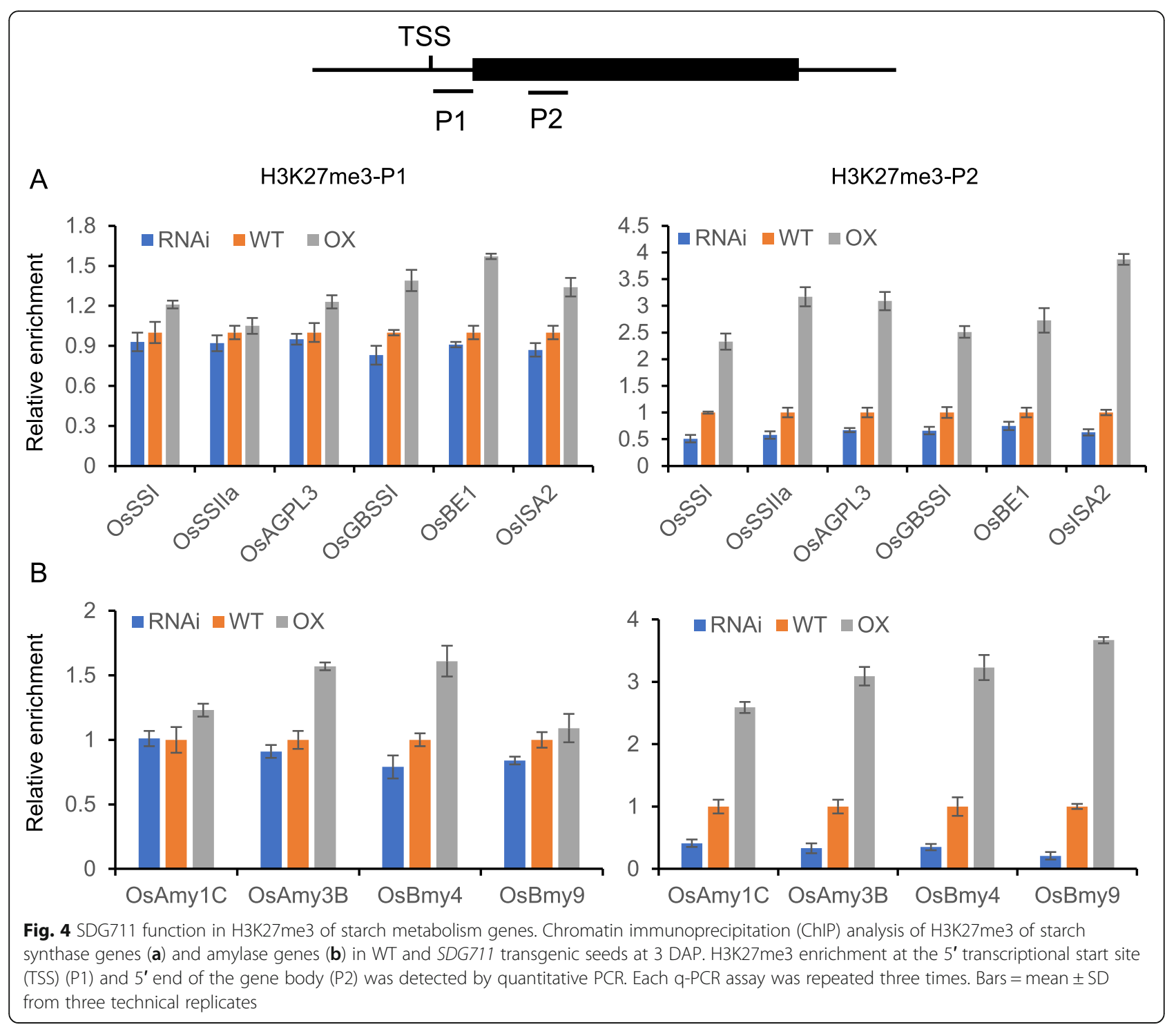

plants. Although the expression levels of other genes in the corresponding transgenic plants changed, the enrichment of H3K27me3 did not change significantly (Figure S5). This may support the indirect effect of SDG711 on their expression. These data suggest that SDG711mediated H3K27me3 is involved in the regulation of starch synthesis and degradation related genes during seed development.

\section{SDG711 Directly Binds to Starch Synthesis Genes and Amylase Genes}

To further assess the function of SDG711 on the regulation of starch synthesis genes and amylase genes, we performed anti-SDG711 ChIP assays and analyzed them by real-time PCR using the same primer sets as for the histone methylation ChIP. Non-immunized serum was used as a control. The analysis revealed that SDG711 was obviously enhanced in the gene body of six starch synthesis genes (OsSSI, OsSSIIa, OsAGPL3, OsGBSSI, $O s B E I$, and OsISA2) and four starch amylase genes (OsA$m y 1 C$, OsAmy3B, OsBmy4, and OsBmy9) compared with the control (Fig. 5). These results suggest that SDG711 may directly target these genes.

\section{H3K27me3 Can Affect H3K4me3 and H3K9ac on some Starch Synthesis Genes and Amylase Genes}

Because H3K27me3 is antagonistic to H3K4me3 in terms of gene activity and $\mathrm{H} 3 \mathrm{~K} 9 \mathrm{ac}$ has also been reported to be enriched in these genes, we analyzed whether alteration of H3K27me3 affected H3K4me3 and $\mathrm{H} 3 \mathrm{~K} 9 \mathrm{ac}$ on the starch synthesis genes and amylase genes in the transgenic plants. We performed anti-H3K4me3 and H3K9ac ChIP assays and analysis by real-time PCR using the same primer sets as for H3K27me3 ChIP. 

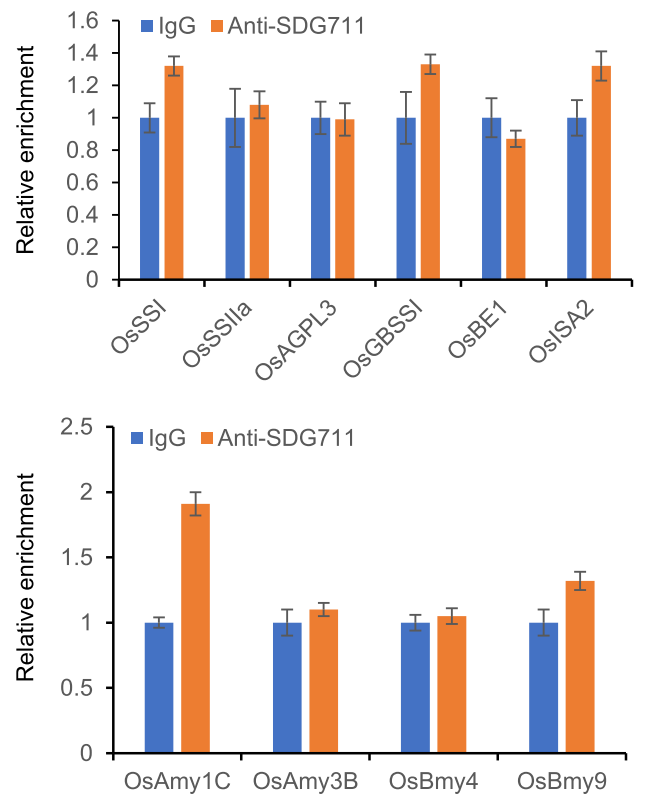
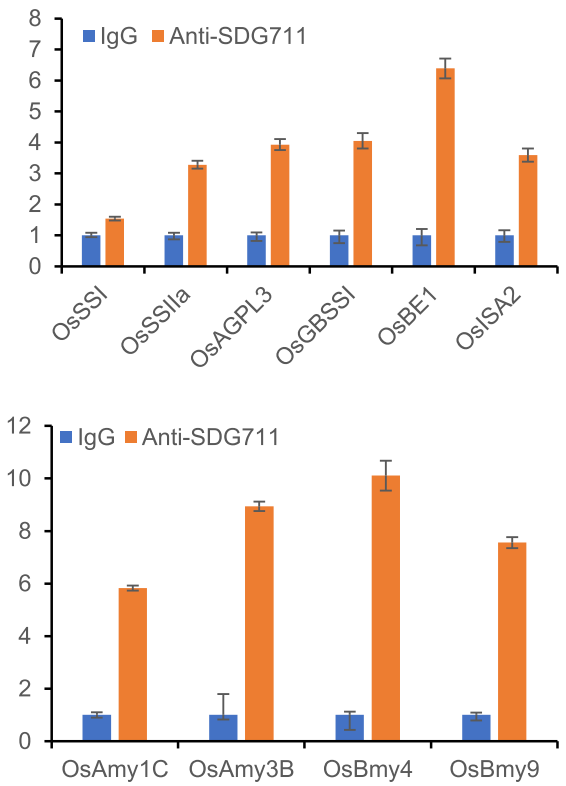

Fig. 5 Direct association of SDG711 protein with genes of starch metabolism. SDG711 protein enrichment of the starch metabolism genes in WT seeds at 3 DAP tested by ChIP with anti-SDG711. Non-immunized serum (lgG) was used as a control. SDG711 protein enrichment at the 5' transcriptional start site (TSS) (P1) and $5^{\prime}$ end of the gene body (P2) was detected by quantitative PCR (q-PCR). Each q-PCR assay was repeated three times. Values are the mean \pm SD from three technical replicates

Enrichment for H3K4me3 and H3K9ac occurs near the 5' transcriptional start site (TSS), and the P1 primers were appropriate. The analysis revealed there were relatively higher levels of $\mathrm{H} 3 \mathrm{~K} 4 \mathrm{me} 3$ on OsAGPL3, $O s B E I$, and OSISA2b in SDG711 RNAi plants, and relatively lower levels in $S D G 711$ overexpression plants than in WT plants (Fig. 6a). This inversely correlated with the results for $\mathrm{H} 3 \mathrm{~K} 27 \mathrm{me} 3$, suggesting that SDG711-mediated H3K27me3 might affect H3K4me3 at these three loci. Similarly, H3K9ac on OsSSI, OsSSIIa, OsBmy4, and OsBmy9 was increased in SDG711 RNAi plants but decreased in SDG711 overexpression plants (Fig. 6b), which also inversely correlated with the results for H3K27me3, suggesting that SDG711-mediated H3K27me3 might affect H3K9ac at these five loci. Although the alteration of $\mathrm{H} 3 \mathrm{~K} 27 \mathrm{me} 3$ is relatively obvious in other genes, the enrichment of H3K4me3 or H3K9ac on these genes did not change significantly in the transgenic plants.

In addition, We validated the presence of H3K4me3H3K27me3 and H3K9ac-H3K27me3 bivalent chromatin state on some starch metabolism genes by re-ChIP (Fig. 7). The analysis revealed that there are H3K4me3H3K27me3 bivalent chromatin state on OsAGPL3, $O s B E I$, and OsISA2b (Fig. 7a), and there are H3K9acH3K27me3 bivalent chromatin state on OsSSI, OsSSIIa, OsBmy4, and OsBmy9 (Fig. 7b).
These results indicate that both single and multiple modifications can regulate gene expression, and that the multiple modifications of starch synthesis genes and amylase genes are finely balanced. Disruption of this balance causes abnormal gene expression (Figure S9).

\section{Discussion}

In Arabidopsis, at least three PRC2-like complexes, the EMBRYONIC FLOWER (EMF), VERNALIZATION (VRN), and FERTILISATION INDEPENDENT SEED (FIS) complexes, play critical roles in different developmental stages. The EMF complex (CLF/SWN, EMF2, FIE and MSI1) mainly promotes vegetative development of the plant, and delays reproduction. The VRN complex (CLF/SWN, VRN2, FIE and MSI1) establishes epigenetic silencing of FLC after vernalization and enables flowering. The FIS complex (MEA, SWN, FIS2, FIE and MSI1) prevents seed development in the absence of fertilization and is required for normal seed development (Hennig and Derkacheva 2009). Although there have been some advances in the studies on the components of the PRC2 complex in rice (Chen et al. 2017; Folsom et al. 2014; Huang et al. 2016; Li et al. 2014; Liu et al. 2016; Liu et al. 2014b; Liu et al. 2015; Nallamilli et al. 2013; Zhong et al. 2018), the specific composition and diversity of the PRC2 complex have not been clearly described. Combining the results of previous research and our current 
A

H3K4me3-P1

H3K4me3-P2
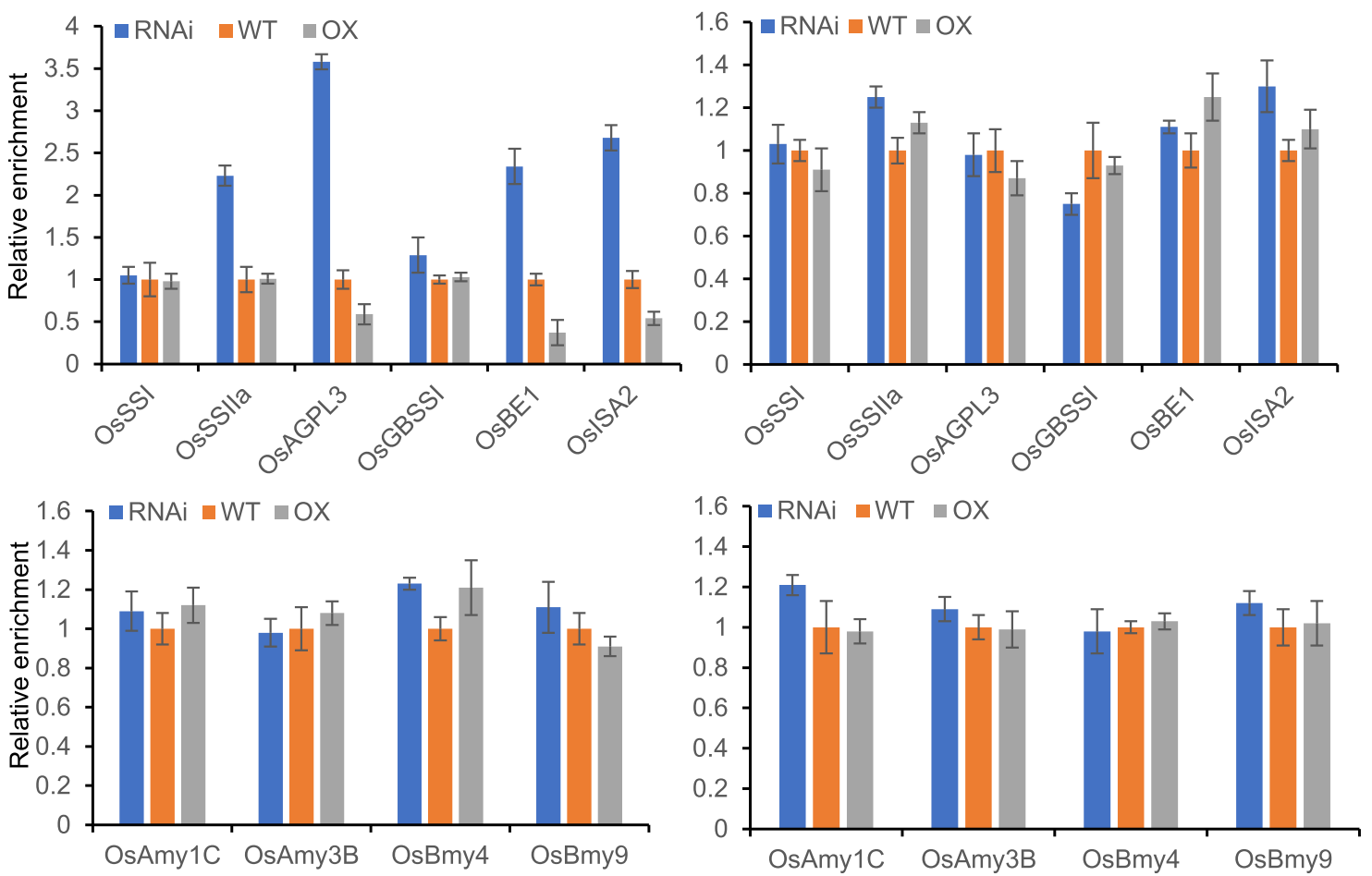

B

H3K9ac-P1
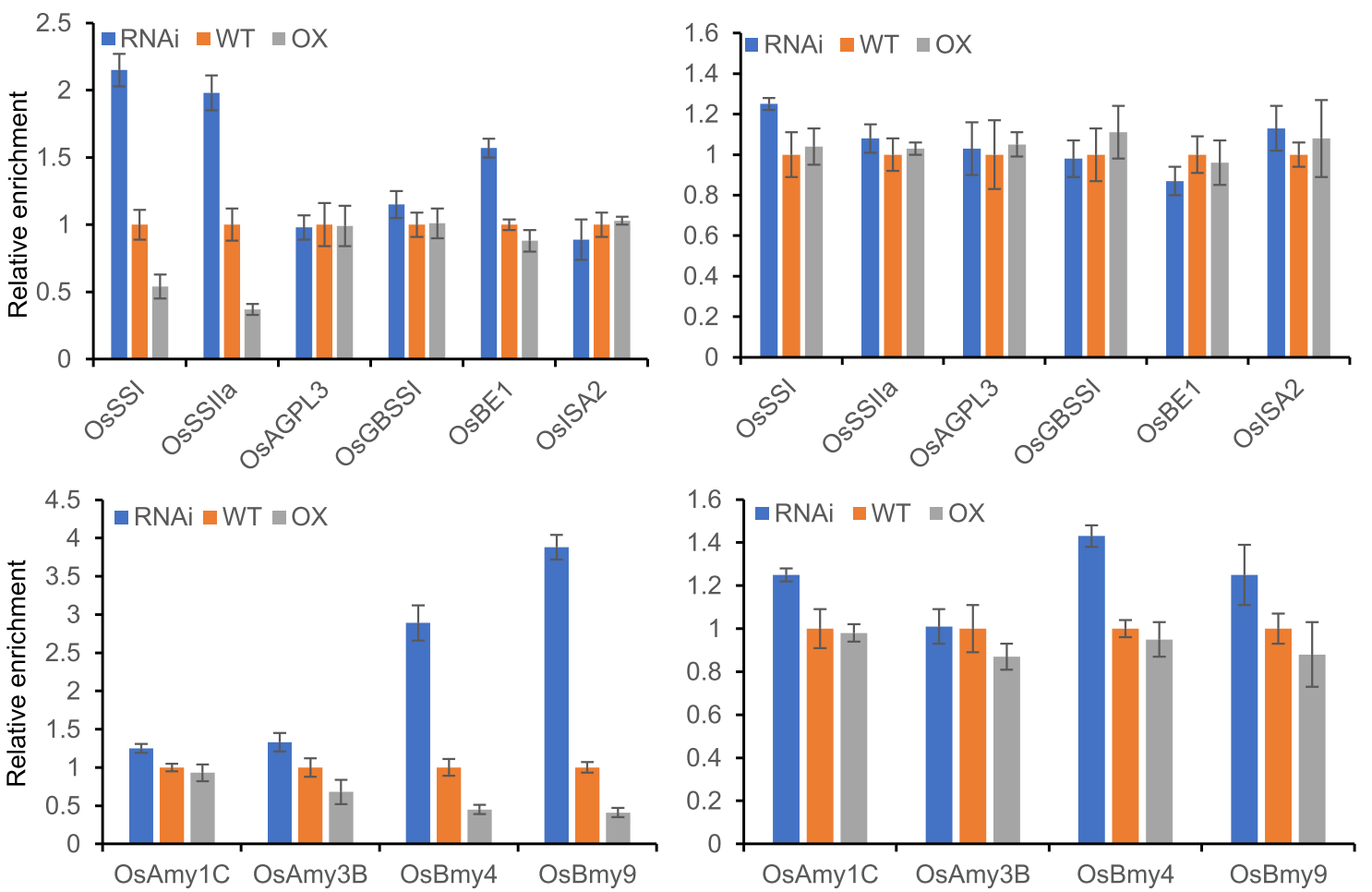

Fig. 6 (See legend on next page.) 
(See figure on previous page.)

Fig. 6 H3K4me3 and H3K9ac enrichments of starch metabolism genes. Chromatin immunoprecipitation (ChIP) analysis of H3K4me3 (a) and H3K9ac (b) of starch synthase genes and amylase genes in WT and SDG711 transgenic seeds at 3 DAP. H3K4me3 and H3K9ac enrichment at the $5^{\prime}$ transcriptional start site (TSS) (P1) and the $5^{\prime}$ end of the gene body (P2) were detected by quantitative PCR (q-PCR). Each q-PCR assay was repeated three times. Bars $=$ mean \pm SD from three technical replicates

study on the relationship between some key components (Figure S6), we propose that during the flowering stage of rice, there are two PRC2 complexes, OsCLF (SDG711)complex and OsiZE1 (SDG718)-complex, regulating the flowering time under long daylight and short daylight, respectively. During the other development stages, the OsFIE1-complex mainly plays roles in seed development and the OsFIE2-complex plays essential roles in the regulation of rice vegetative and reproductive development (Figure S7).

MEA and FIS2 are the two core components of PRC2. It was revealed by previous studies that they are imprinted genes and only transcribed from the maternal
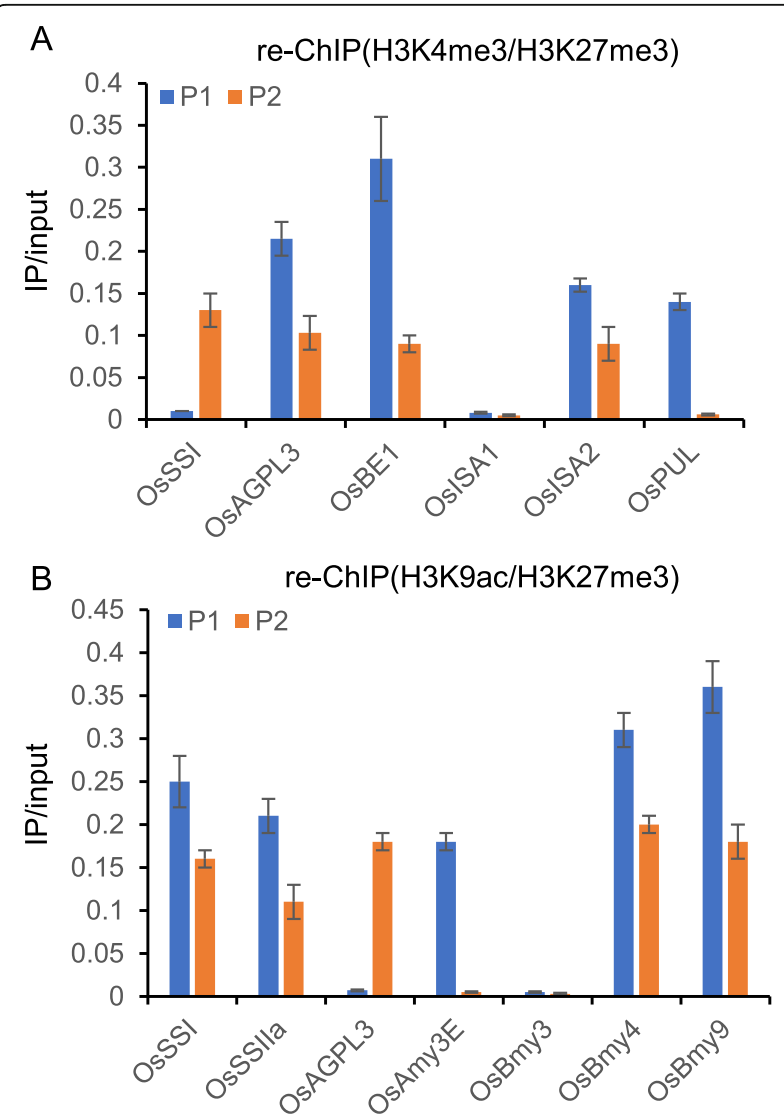

Fig. $7 \mathrm{H} 3 \mathrm{~K} 4 \mathrm{me} 3-\mathrm{H} 3 \mathrm{~K} 27 \mathrm{me} 3$ and H3K9ac-H3K27me3 bivalent chromatin state on some starch metabolism genes. Re-ChIP analysis of H3K4me3-H3K27me3 (a) and H3K9ac-H3K27me3 (b) of starch synthase genes and amylase genes in WT seeds at 3 DAP. H3K4me3 and H3K9ac enrichment an the $5^{\prime}$ transcriptional start site (TSS) (P1) and H3K27me3 enrichment at $5^{\prime}$ end of the gene body (P2) were detected by quantitative. Each q-PCR assay was repeated three times. Bars $=$ mean \pm SD from three technical replicates allele in the endosperm and interact directly with each other to regulate endosperm formation by controlling the activity of a number of imprinted genes in the endosperm in Arabidopsis (Kinoshita et al. 1999; Luo et al. 2000; Spillane et al. 2000; Baroux et al. 2006). In rice, OsFIE1, the homolog of FIS2 expressed only in the endosperm, is a maternally expressed imprinting gene. The other five PRC2 genes (OsFIE2, OsEMF1, OsEMF2, $O s C L F$ and OsiZE1) are expressed in a wide range of tissues and are not imprinted (Luo et al. 2009). However, recent research showed that except for OsCLF (SDG711) being a non-imprinted gene, four other genes that were considered as non-imprinted genes showed the characteristics of imprinted genes at different stages of endosperm development in rice (Kuang et al. 2019). In addition, our recent research suggested that the CLF homologous gene OsCLF (SDG711) regulates endosperm development by directly binding to the gene body region of several starch synthesis genes and amylase genes to mediate H3K27me3 enrichment in rice. Meanwhile, we tested the expression of some imprinted genes surveyed previously (Luo et al. 2011; Rodrigues et al. 2013; Yuan et al. 2017) in SDG711 overexpression plants. With the overexpression of SDG711, the expression of these genes increased or decreased, which indicates that SDG711 may also be able to regulate the development of endosperm by affecting the expression of these imprinted genes (Figure S8). Overall, although the PRC2 genes are highly conserved and play an important role in the entire growth and development stage of plants, the components, and functions of PRC2 are very different between Arabidopsis and rice such as the imprinting effect and their roles in regulating endosperm development.

The N-terminal tails of the core histones undergo multiple covalent modifications. The histone code hypothesis suggests that multiple histone modifications act in a combinatorial manner to affect gene transcription (Strahl and Allis 2000; Schreiber and Bernstein 2002). As a combination of dense marks in short clusters, situated at strategic locations in the histones, the "modification cassette" was proposed to clarify the mechanism that may control the biological reading of different modification patterns (Fischle et al. 2003). The modification cassette also indicated that there are multiple modifications on the same nucleosome to coordinately regulate the expression of the same gene. For example, the crosstalk between serine phosphorylation and lysine 
methylation of the mitochondrial protein DAM1 and the transcription factor p53 has been described in human cancer cells (Fischle et al. 2003; Zhang and Dent 2005). In Arabidopsis, the regulation of the FLC locus provides a plant model of how multiple chromatin-modifying systems have emerged as important components in the control of a major developmental switch, the transition to flowering. H3K4me3 and histone acetylation are associated with active FLC expression, whereas histone deacetylation and H3K9me2 and H3K27me3 are involved in FLC repression (He and Amasino 2005).

Subsequently, the regions harboring both repressive and active chromatin modifications were defined as bivalent domains (Bernstein et al. 2006). The bivalent domain (BD) marked by H3K27me3 and H3K4me3 which are catalyzed by specific PcG and TrxG complexes, respectively, was first discovered and characterized in mouse embryonic stem cells (ESCs) (Bernstein et al. 2006). The co-occurrence of H3K4me3 and H3K27me3 is often found in promoter regions of developmentally expressed TFs and developmental genes to be rapidly switched on during differentiation in specific cell types in mouse ESCs and human ESCs (Zhao et al. 2007; Bernstein et al. 2006; Xiang et al. 2020). In addition to the bivalent promoter described above, there is another class of bivalent region called a bivalent enhancer (Blanco et al. 2020). It has been shown that the co-occurrence of H3K4me1 and H3K27me3 marks the presence of bivalent enhancers in hESCs and mESCs. They likely play a key role during differentiation, similar to bivalent promoters (Rada-Iglesias et al. 2011; Zentner et al. 2011). Recent studies have shown that DNA methylation can affect the H327me3: H3K4me3 ratio of the bivalent promoter in different cell types, and DNA hypermethylation of the bivalent promoter in cancer is related to the H3K27me3: H3K4me3 ratio in embryonic stem cells (Dunican et al. 2020). In plants, there are also a few studies describing the existence of divalent modifications (H3K4me3/ H3K27me3) during the development process and stress treatment (Berr et al. 2010; Liu et al. 2014a; Sequeira-Mendes et al. 2014; Zeng et al. 2019).

Modification cassettes and bivalent modifications indicate that histone modifications play a very important role in the development of organisms, and these modifications have balanced relationships. When the balance of these modifications is disrupted, the growth and development of individual plants may be seriously affected. This study also pointed out that during the development of rice endosperm starch, some key genes might be coordinately regulated by multiple histone modifications. For example, some starch metabolism-related genes are regulated by $\mathrm{H} 3 \mathrm{~K} 27 \mathrm{me} 3$ and $\mathrm{H} 3 \mathrm{~K} 4 \mathrm{me} 3$, and some are regulated by $\mathrm{H} 3 \mathrm{~K} 27 \mathrm{me} 3$ and $\mathrm{H} 3 \mathrm{~K} 9 \mathrm{ac}$. Under normal circumstances, these modifications will be in a balanced state. Once a certain modification changes, the balance will be broken and the gene expression will be abnormal (Figure S9). This indicates that gene expression is regulated by multiple modifications in many cases, and this regulation might be very precise.

\section{Conclusions}

Collectively, our results show that overexpression and downregulation of SDG711 leads to a decrease and increase in the expression level of genes related to starch accumulation, resulting in smaller seeds or even seed abortion. The ChIP assay showed that SDG711-mediated H3K27me3 changed significantly in genes related to endosperm development, and SDG711 can directly bind to the gene body region of several starch synthesis genes and amylase genes. In addition, H3K4me3 and H3K9ac modifications also cooperate with H3K27me3 to regulate the development of the endosperm. Our results suggest that the crosstalk between SDG711-mediated H3K27me3 and H3K4me3, and H3K9ac are involved in starch accumulation to control normal seed development. Our work provides new insights into the regulation of endosperm development.

\section{Supplementary Information}

The online version contains supplementary material available at https://doi. org/10.1186/s12284-021-00467-y.

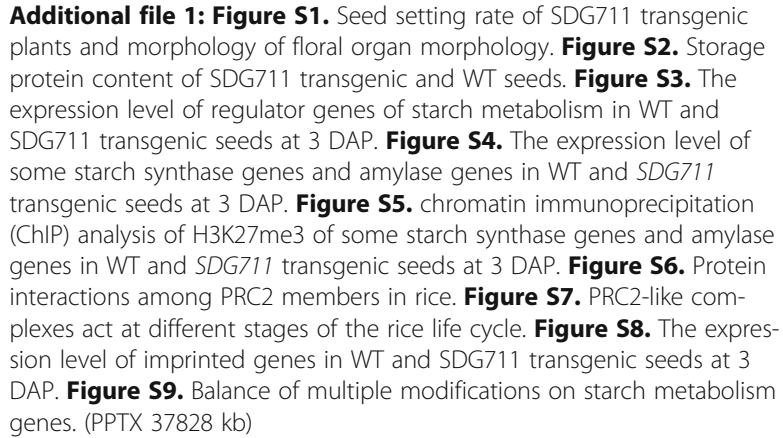

Additional file 1: Figure S1. Seed setting rate of SDG711 transgenic plants and morphology of floral organ morphology. Figure S2. Storage protein content of SDG711 transgenic and WT seeds. Figure S3. The expression level of regulator genes of starch metabolism in WT and SDG711 transgenic seeds at 3 DAP. Figure S4. The expression level of some starch synthase genes and amylase genes in WT and SDG711 transgenic seeds at 3 DAP. Figure S5. chromatin immunoprecipitation (ChIP) analysis of H3K27me3 of some starch synthase genes and amylase genes in WT and SDG711 transgenic seeds at 3 DAP. Figure S6. Protein interactions among PRC2 members in rice. Figure S7. PRC2-like complexes act at different stages of the rice life cycle. Figure S8. The expression level of imprinted genes in WT and SDG711 transgenic seeds at 3 DAP. Figure S9. Balance of multiple modifications on starch metabolism genes. (PPTX $37828 \mathrm{~kb}$ )

Additional file 2: Table S1. Oligonucleotides primers used in the study.

\section{Abbreviations}

H3K27me3: H3 lysine 27 trimethylation; H3K4me3: H3 lysine 27 trimethylation; H3K9ac: H3 lysine 9 acetylation; PCG: Polycomb group; OsAmy: a-amylase; OsBmy: $\beta$-amylase; ds: RNAi plants of SDG711; OX: Overexpression plants of SDG711; WT: Wildtype; DAP: Day After Pollination; ACC: Apparent Amylase Content; ChIP: Chromatin immunoprecipitation assay; qRT-PCR: Quantitative Reverse Transcription Polymerase Chain Reaction

\section{Acknowledgements}

We thank Prof. Dao-Xiu Zhou and Prof. Yu Zhao (National Key Laboratory of Crop Genetics Improvement, Huazhong Agricultural University, Wuhan,

China) for their suggestions to this article.

\section{Authors' Contributions}

$X \mathrm{~L}$ and $\mathrm{CZ}$ designed the research; $\mathrm{XL}, J \mathrm{~L}, \mathrm{TL}, \mathrm{HY}$, and $\mathrm{PW}$ conducted the experiments; $J$, LS, YZ and CB analyzed the data; $X L$ wrote the article; $C Z$ supervised and complemented the manuscript. The authors read and approved the manuscript. 


\section{Funding}

This study was supported by the Discipline Innovation Team Foundation of Jianghan University (03100074), National Natural Science Foundation of China (NSFC31600981, NSFC 31501028, and NSFC31701100).

\section{Availability of Data and Materials}

The data sets supporting the conclusions of this article are included within the article and its additional files.

\section{Ethics Approval and Consent to Participate \\ Not applicable.}

\section{Consent for Publication}

Not applicable.

\section{Competing Interests}

The authors declare that they have no competing interests.

\section{Author details}

${ }^{1}$ Institute for Interdisciplinary Research, Jianghan University, Wuhan 430056, China. ${ }^{2}$ Key Laboratory of Three Gorges Regional Plant Genetics \& Germplasm Enhancement (CTGU) /Biotechnology Research Center, China Three Gorges University, Yichang 443002, China. ${ }^{3}$ Key Laboratory of Biology and Genetic Improvement of Oil Crops, the Ministry of Agriculture and Rural Affairs, Oil Crops Research Institute, Chinese Academy of Agricultural Sciences, Wuhan 430062, China. ${ }^{4}$ Institute for Systems Biology, Jianghan University, Wuhan 430056, Hubei, China.

Received: 2 July 2020 Accepted: 17 February 2021

Published online: 05 March 2021

\section{References}

Asatsuma S, Sawada C, Kitajima A, Asakura T, Mitsui T (2006) a-Amylase affects starch accumulation in Rice grains. J Appl Glycosci 53(3):187-192

Baroux C, Gagliardini V, Page DR, Grossniklaus U (2006) Dynamic regulatory interactions of Polycomb group genes: MEDEA autoregulation is required for imprinted gene expression in Arabidopsis. Genes Dev 20(9):1081-1086. https://doi.org/10.1101/gad.378106

Bernstein BE, Mikkelsen TS, Xie X, Kamal M, Huebert DJ, Cuff J, Fry B, Meissner A, Wernig M, Plath K, Jaenisch R, Wagschal A, Feil R, Schreiber SL, Lander ES (2006) A bivalent chromatin structure marks key developmental genes in embryonic stem cells. Cell 125(2):315-326. https://doi.org/10.1016/j.cell.2006. 02.041

Berr A, McCallum EJ, Menard R, Meyer D, Fuchs J, Dong A, Shen WH (2010) Arabidopsis SET DOMAIN GROUP2 is required for H3K4 trimethylation and is crucial for both sporophyte and gametophyte development. Plant Cell 22(10):3232-3248. https://doi.org/10.1105/tpc.110.079962

Blanco E, Gonzalez-Ramirez M, Alcaine-Colet A, Aranda S, Di Croce L (2020) The bivalent genome: characterization, structure, and regulation. Trends in genetics: TIG 36(2):118-131. https://doi.org/10.1016/j.tig.2019.11.004

Charron JB, He H, Elling AA, Deng XW (2009) Dynamic landscapes of four histone modifications during deetiolation in Arabidopsis. Plant Cell 21(12):3732-3748. https://doi.org/10.1105/tpc.109.066845

Chen M, Xie S, Ouyang Y, Yao J (2017) Rice PCG gene OsEMF2b controls seed dormancy and seedling growth by regulating the expression of OsVP1. Plant Science 260:80-89. https://doi.org/10.1016/j.plantsci.2017.04.005

Czermin B, Melfi R, McCabe D, Seitz V, Pirrotta V (2002) Drosophila enhancer of Zeste/ESC complexes have a histone $\mathrm{H} 3$ methyltransferase activity that marks chromosomal Polycomb sites. Cell 111(2):185-196

Dunican DS, Mjoseng HK, Duthie L, Flyamer IM, Bickmore WA, Meehan RR (2020) Bivalent promoter hypermethylation in cancer is linked to the H327me3/ H3K4me3 ratio in embryonic stem cells. BMC Biol 18(1):25. https://doi.org/1 $0.1186 /$ s12915-020-0752-3

Fischle W, Wang Y, Allis CD (2003) Binary switches and modification cassettes in histone biology and beyond. Nature 425(6957):475-479

Folsom JJ, Begcy K, Hao X, Wang D, Walia H (2014) Rice fertilization-independent Endosperm 1 regulates seed size under heat stress by controlling early endosperm development. Plant Physiol 165(1):238-248. https://doi.org/10.11 04/pp.113.232413
Fu FF, Xue HW (2010) Coexpression analysis identifies Rice starch Regulator1, a rice AP2/EREBP family transcription factor, as a novel rice starch biosynthesis regulator. Plant Physiol 154(2):927-938. https://doi.org/10.1104/pp.110.159517

Ge G, Zheng X, Wu J, Ye Z, Shi C (2007) Analysis of the conditional correlations from different genetic systems between the protein content and the appearance quality traits of indica rice. Journal of Genetics and Genomics 34(2):129-137

He G, Elling AA, Deng XW (2011) The epigenome and plant development. Annu Rev Plant Biol 62:411-435. https://doi.org/10.1146/annurev-arplant-042110-1 03806

He Y, Amasino RM (2005) Role of chromatin modification in flowering-time control. Trends Plant Sci 10(1):30-35. https://doi.org/10.1016/j.tplants.2004.11.003

Hennig L, Derkacheva M (2009) Diversity of Polycomb group complexes in plants: same rules, different players? Trends in genetics: TIG 25(9):414-423. https://doi.org/10.1016/j.tig.2009.07.002

Hu Y, Liu D, Zhong X, Zhang C, Zhang Q, Zhou DX (2012) CHD3 protein recognizes and regulates methylated histone $\mathrm{H} 3$ lysines 4 and 27 over a subset of targets in the rice genome. Proc Natl Acad Sci U S A 109(15):57735778. https://doi.org/10.1073/pnas.1203148109

Huang X, Lu Z, Wang X, Ouyang Y, Chen W, Xie K, Wang D, Luo M, Luo J, Yao J (2016) Imprinted gene OsFIE1 modulates rice seed development by influencing nutrient metabolism and modifying genome H3K27me3. Plant J 87(3):305-317. https://doi.org/10.1111/tpj.13202

Juliano B (1971) A simplified assay for milled rice amylose. Cereal Sci Today 16: 334-360

Kinoshita T, Yadegari R, Harada JJ, Goldberg RB, Fischer RL (1999) Imprinting of the MEDEA polycomb gene in the Arabidopsis endosperm. Plant Cell 11(10): 1945-1952

Kuang Q, Wang Y, Li S (2019) Detailed observation on expression dynamics of Polycomb group genes during rice early endosperm development in subspecies hybridization reveals their characteristics of parent-of-origin genes. Rice 12(1):64. https://doi.org/10.1186/s12284-019-0306-X

Li S, Zhou B, Peng X, Kuang Q, Huang X, Yao J, Du B, Sun MX (2014) OsFIE2 plays an essential role in the regulation of rice vegetative and reproductive development. New phytologist 201(1):66-79. https://doi. org/10.1111/nph.12472

Liu N, Fromm M, Avramova Z (2014a) H3K27me3 and H3K4me3 chromatin environment at super-induced dehydration stress memory genes of Arabidopsis thaliana. Mol Plant 7(3):502-513. https://doi.org/10.1093/ $\mathrm{mp} / \mathrm{ssu} 001$

Liu X, Wei X, Sheng Z, Jiao G, Tang S, Luo J, Hu P (2016) Polycomb protein OsFIE2 affects plant height and grain yield in Rice. PLoS One 11(10): e0164748. https://doi.org/10.1371/journal.pone.0164748

Liu X, Zhou C, Zhao Y, Zhou S, Wang W, Zhou DX (2014b) The rice enhancer of zeste $[E(Z)]$ genes SDG711 and SDG718 are respectively involved in long day and short day signaling to mediate the accurate photoperiod control of flowering time. Front Plant Sci 5:591. https://doi.org/10.3389/fpls.2014.00591

Liu X, Zhou S, Wang W, Ye Y, Zhao Y, Xu Q, Zhou C, Tan F, Cheng S, Zhou DX (2015) Regulation of histone methylation and reprogramming of gene expression in the rice inflorescence meristem. Plant Cell 27(5):1428-1444. https://doi.org/10.1105/tpc.15.00201

Livak KJ, Schmittgen TD (2001) Analysis of relative gene expression data using real-time quantitative $P C R$ and the $2-\Delta \Delta C T$ method. Methods 25(4):402-408. https://doi.org/10.1006/meth.2001.1262

Luo M, Bilodeau P, Dennis ES, Peacock WJ, Chaudhury A (2000) Expression and parent-of-origin effects for FIS2, MEA, and FIE in the endosperm and embryo of developing Arabidopsis seeds. Proc Natl Acad Sci U S A 97(19):1063710642. https://doi.org/10.1073/pnas.170292997

Luo M, Platten D, Chaudhury A, Peacock WJ, Dennis ES (2009) Expression, imprinting, and evolution of rice homologs of the polycomb group genes. Mol Plant 2(4):711-723. https://doi.org/10.1093/mp/ssp036

Luo M, Taylor JM, Spriggs A, Zhang H, Wu X, Russell S, Singh M, Koltunow A (2011) A genome-wide survey of imprinted genes in rice seeds reveals imprinting primarily occurs in the endosperm. PLoS Genet 7(6):e1002125. https://doi.org/10.1371/journal.pgen.1002125

Makarevitch I, Eichten SR, Briskine R, Waters AJ, Danilevskaya ON, Meeley RB, Myers CL, Vaughn MW, Springer NM (2013) Genomic distribution of maize facultative heterochromatin marked by trimethylation of H3K27. Plant Cell 25(3):780-793. https://doi.org/10.1105/tpc.112.106427

Nallamilli BR, Zhang J, Mujahid H, Malone BM, Bridges SM, Peng Z (2013) Polycomb group gene OsFIE2 regulates rice (Oryza sativa) seed development 
and grain filling via a mechanism distinct from Arabidopsis. PLoS Genet 9(3): e1003322. https://doi.org/10.1371/journal.pgen.1003322

Ohdan T, Francisco PB Jr, Sawada T, Hirose T, Terao T, Satoh H, Nakamura Y (2005) Expression profiling of genes involved in starch synthesis in sink and source organs of rice. J Exp Bot 56(422):3229-3244. https://doi.org/10.1093/ jxb/eri292

Peng C, Wang Y, Liu F, Ren Y, Zhou K, LV J, Zheng M, Zhao S, Zhang L, Wang C, Jiang L, Zhang X, Guo X, Bao Y, Wan J (2014) FLOURY ENDOSPERM6 encodes a CBM48 domain-containing protein involved in compound granule formation and starch synthesis in rice ENDOSPERM. Plant J 77(6):917-930. https://doi.org/10.1111/tpj.12444

Qian S, Lv X, Scheid RN, Lu L, Yang Z, Chen W, Liu R, Boersma MD, Denu JM, Zhong X, Du J (2018) Dual recognition of H3K4me3 and H3K27me3 by a plant histone reader SHL. Nat Commun 9(1):2425. https://doi.org/10.1038/ s41467-018-04836-y

Rada-Iglesias A, Bajpai R, Swigut T, Brugmann SA, Flynn RA, Wysocka J (2011) A unique chromatin signature uncovers early developmental enhancers in humans. Nature 470(7333):279-283. https://doi.org/10.1038/nature09692

Rodrigues JA, Ruan R, Nishimura T, Sharma MK, Sharma R, Ronald PC, Zilberman D (2013) Imprinted expression of genes and small RNA is associated with localized hypomethylation of the maternal genome in rice endosperm. Proc Natl Acad Sci 110(19):7934-7939

Schmidt R, Schippers JH, Mieulet D, Watanabe M, Hoefgen R, Guiderdoni E, Mueller-Roeber B (2014) SALT-RESPONSIVE ERF1 is a negative regulator of grain filling and gibberellin-mediated seedling establishment in rice. Mol Plant 7(2):404-421. https://doi.org/10.1093/mp/sst131

Schreiber SL, Bernstein BE (2002) Signaling network model of chromatin. Cell 111(6):771-778

Schuettengruber B, Cavalli G (2009) Recruitment of polycomb group complexes and their role in the dynamic regulation of cell fate choice. Development 136(21):3531-3542. https://doi.org/10.1242/dev.033902

Sequeira-Mendes J, Araguez I, Peiro R, Mendez-Giraldez R, Zhang X, Jacobsen SE, Bastolla U, Gutierrez C (2014) The functional topography of the Arabidopsis genome is organized in a reduced number of linear motifs of chromatin states. Plant Cell 26(6):2351-2366. https://doi.org/10.1105/tpc.114.124578

She KC, Kusano H, Koizumi K, Yamakawa H, Hakata M, Imamura T, Fukuda M, Naito N, Tsurumaki Y, Yaeshima M, Tsuge T, Matsumoto K, Kudoh M, Itoh E, Kikuchi S, Kishimoto N, Yazaki J, Ando T, Yano M, Aoyama T, Sasaki T, Satoh H, Shimada H (2010) A novel factor FLOURY ENDOSPERM2 is involved in regulation of rice grain size and starch quality. Plant Cell 22(10):3280-3294. https://doi.org/10.1105/tpc.109.070821

Spillane C, MacDougall C, Stock C, Köhler C, Vielle-Calzada J, Nunes SM, Grossniklaus U, Goodrich J (2000) Interaction of the Arabidopsis Polycomb group proteins FIE and MEA mediates their common phenotypes. Curr Biol 10(23):1535-1538

Steffen PA, Ringrose $L$ (2014) What are memories made of? How Polycomb and Trithorax proteins mediate epigenetic memory. Nat Rev Mol Cell Biol 15(5): 340-356. https://doi.org/10.1038/nrm3789

Strahl BD, Allis CD (2000) The language of covalent histone modifications. Nature 403(6765):41-45

Turner DH, Turner J (1960) The hydrolysis of glucose monophosphates by a phosphatase preparation from pea seeds. Biochem J 74(3):486

Wang JC, Xu H, Zhu Y, Liu QQ, Cai XL (2013) OsbZIP58, a basic leucine zipper transcription factor, regulates starch biosynthesis in rice endosperm. J Exp Bot 64(11):3453-3466. https://doi.org/10.1093/jxb/ert187

Xiang Y, Zhang Y, Xu Q, Zhou C, Liu B, Du Z, Zhang K, Zhang B, Wang X, Gayen S, Liu L, Wang Y, Li Y, Wang Q, Kalantry S, Li L, Xie W (2020) Epigenomic analysis of gastrulation identifies a unique chromatin state for primed pluripotency. Nat Genet 52(1):95-105. https://doi.org/10.1038/s41588-019-0545-1

Xing MQ, Zhang YJ, Zhou SR, Hu WY, Wu XT, Ye YJ, Wu XX, Xiao YP, Li X, Xue HW (2015) Global analysis reveals the crucial roles of DNA methylation during Rice seed development. Plant Physiol 168(4):1417-1432. https://doi. org/10.1104/pp.15.00414

Yang Z, Qian S, Scheid RN, Lu L, Chen X, Liu R, Du X, Lv X, Boersma MD, Scalf M, Smith LM, Denu JM, Du J, Zhong $X$ (2018) EBS is a bivalent histone reader that regulates floral phase transition in Arabidopsis. Nat Genet 50(9):12471253. https://doi.org/10.1038/s41588-018-0187-8

Yuan J, Chen S, Jiao W, Wang L, Wang L, Ye W, Lu J, Hong D, You S, Cheng Z, Yang DL, Chen ZJ (2017) Both maternally and paternally imprinted genes regulate seed development in rice. The New phytologist 216(2):373-387. https://doi.org/10.1111/nph.14510
Zeng Z, Zhang W, Marand AP, Zhu B, Buell CR, Jiang J (2019) Cold stress induces enhanced chromatin accessibility and bivalent histone modifications H3K4me3 and H3K27me3 of active genes in potato. Genome Biol 20(1):123. https://doi.org/10.1186/s13059-019-1731-2

Zentner GE, Tesar PJ, Scacheri PC (2011) Epigenetic signatures distinguish multiple classes of enhancers with distinct cellular functions. Genome Res 21(8):1273-1283. https://doi.org/10.1101/gr.122382.111

Zhang H, Lu Y, Zhao Y, Zhou DX (2016) OsSRT1 is involved in rice seed development through regulation of starch metabolism gene expression. Plant Sci 248:28-36. https://doi.org/10.1016/j.plantsci.2016.04.004

Zhang K, Dent SY (2005) Histone modifying enzymes and cancer: going beyond histones. J Cell Biochem 96(6):1137-1148. https://doi.org/10.1002/jcb.20615

Zhang X, Bernatavichute W, Cokus S, Pellegrini M, Jacobsen SE (2009) Genomewide analysis of mono-, di- and trimethylation of histone $\mathrm{H} 3$ lysine 4 in Arabidopsis thaliana. Genome Biol 10(6):R62. https://doi.org/10.1186/gb-2 009-10-6-r62

Zhang X, Clarenz O, Cokus S, Bernatavichute W, Pellegrini M, Goodrich J, Jacobsen SE (2007) Whole-genome analysis of histone H3 lysine 27 trimethylation in Arabidopsis. PLoS Biol 5(5):e129. https://doi.org/10.1371/ journal.pbio.0050129

Zhao XD, Han X, Chew JL, Liu J, Chiu KP, Choo A, Orlov YL, Sung WK, Shahab A, Kuznetsov VA, Bourque G, Oh S, Ruan Y, Ng HH, Wei CL (2007) Wholegenome mapping of histone $\mathrm{H} 3$ Lys 4 and 27 trimethylations reveals distinct genomic compartments in human embryonic stem cells. Cell Stem Cell 1(3): 286-298. https://doi.org/10.1016/j.stem.2007.08.004

Zhong J, Peng Z, Peng Q, Cai Q, Peng W, Chen M, Yao J (2018) Regulation of plant height in rice by the Polycomb group genes OsEMF2b, OsFIE2 and OsCLF. Plant Sci 267:157-167. https://doi.org/10.1016/j.plantsci.2017.11.007

Zhu Y, Cai XL, Wang ZY, Hong MM (2003) An interaction between a MYC protein and an EREBP protein is involved in transcriptional regulation of the rice $W_{x}$ gene. J Biol Chem 278(48):47803-47811. https://doi.org/10.1074/jbc.M302 806200

\section{Publisher's Note}

Springer Nature remains neutral with regard to jurisdictional claims in published maps and institutional affiliations.

\section{Submit your manuscript to a SpringerOpen ${ }^{\circ}$ journal and benefit from:}

- Convenient online submission

- Rigorous peer review

- Open access: articles freely available online

- High visibility within the field

- Retaining the copyright to your article

Submit your next manuscript at $\boldsymbol{\nabla}$ springeropen.com 